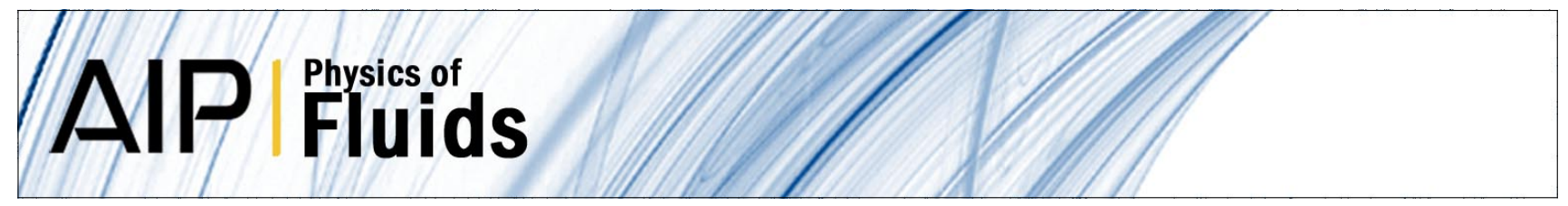

\title{
Finite Rossby radius effects on vortex motion near a gap
}

R. S. Nilawar, E. R. Johnson, and N. R. McDonald

Citation: Phys. Fluids 24, 066601 (2012); doi: 10.1063/1.4721432

View online: http://dx.doi.org/10.1063/1.4721432

View Table of Contents: http://pof.aip.org/resource/1/PHFLE6/v24/i6

Published by the American Institute of Physics.

\section{Related Articles}

Flow mediated interactions between two cylinders at finite Re numbers

Phys. Fluids 24, 043103 (2012)

The continuous spectrum of time-harmonic shear layers

Phys. Fluids 24, 034101 (2012)

The interaction of a vortex ring with a sloped sediment layer: Critical criteria for incipient grain motion

Phys. Fluids 24, 026604 (2012)

Numerical study of flow characteristics behind a stationary circular cylinder with a flapping plate

Phys. Fluids 23, 073601 (2011)

Multimode stretched spiral vortex and nonequilibrium energy spectrum in homogeneous shear flow turbulence Phys. Fluids 23, 035107 (2011)

\section{Additional information on Phys. Fluids}

Journal Homepage: http://pof.aip.org/

Journal Information: http://pof.aip.org/about/about_the_journal

Top downloads: http://pof.aip.org/features/most_downloaded

Information for Authors: http://pof.aip.org/authors

\section{ADVERTISEMENT}

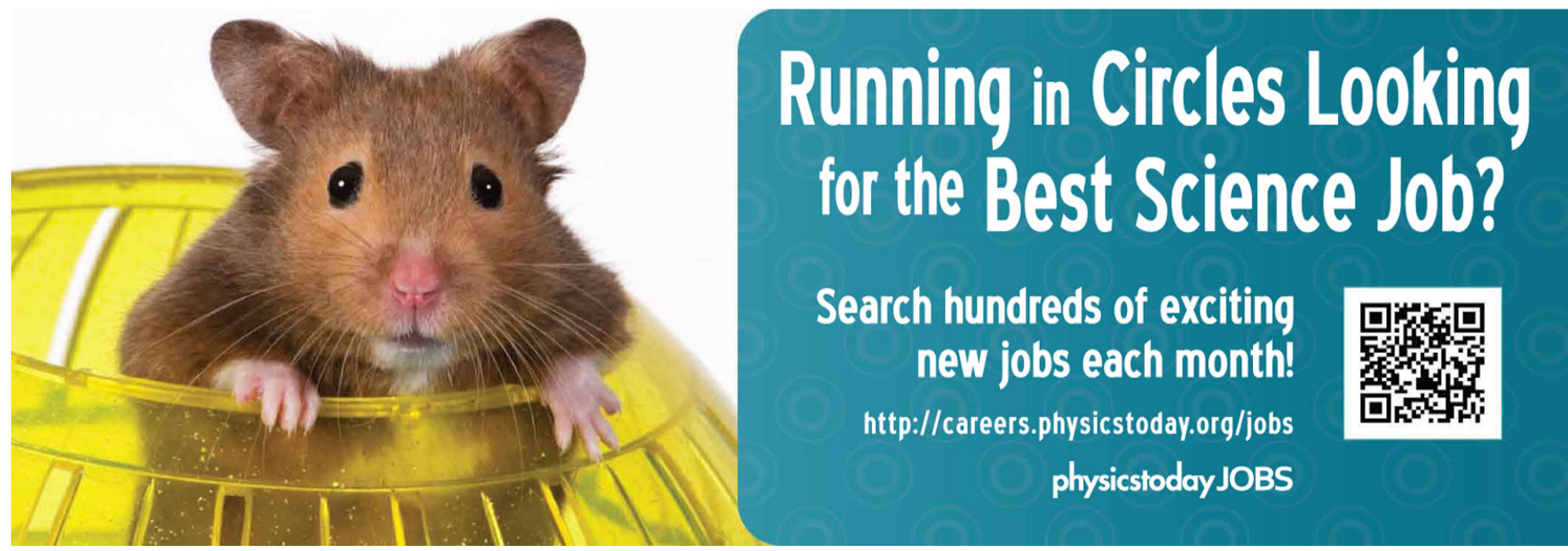




\title{
Finite Rossby radius effects on vortex motion near a gap
}

\author{
R. S. Nilawar, E. R. Johnson, and N. R. McDonald \\ Department of Mathematics, University College London, London WC1E 6BT, \\ United Kingdom
}

(Received 22 November 2011; accepted 29 March 2012; published online 5 June 2012)

\begin{abstract}
This work investigates the effect of the Rossby radius of deformation on the motion of a vortex near a gap in an infinitely long barrier. A key parameter determining the behaviour of the vortex is $a$, the ratio of the Rossby radius of deformation to the width of the gap. Assuming quasi-geostrophic dynamics for a single-layer, reducedgravity fluid, an integral equation is derived whose solution gives the velocity at any point in the fluid. The integral equation is solved numerically and the velocity field is integrated to give the trajectories of point vortices. Combined with the method of contour dynamics, the method can be used to compute the evolution of finite area patches of constant vorticity. The trajectories of point vortices and vortex patches are compared. The patches are initially circular and the centroids of those vortex patches that remain close to circular follow the trajectory and speed of their equivalent point vortices when appropriately normalised. The critical point vortex trajectory (the separatrix) which divides vortices that leap across the gap and those that pass through, is computed for various $a$. Decreasing the Rossby radius of deformation increases the tendency of vortices to pass through the gap. The effect of various background flows on both point vortex and vortex patch motion is also described. [http://dx.doi.org/10.1063/1.4721432]
\end{abstract}

\section{INTRODUCTION}

Long-lived eddies play a significant role in the transport and mixing of ocean properties such as momentum, heat, and salt. For example, meddies ${ }^{1}$ have been tracked for up to several years and carry anomalously salty water from the Mediterranean to the North Atlantic. Such long-lived vortices will inevitably encounter topography in the form of mid-ocean ridges, coastlines, and seamounts. It is of interest to determine how the interaction of vortices with such topographic features affects their transport characteristics.

Vortex interactions with gap-like geometries have been studied in relation to the equatorial currents and eddies in the western Atlantic ${ }^{2}$ showing how fluid can be transported through a gap by eddies. High resolution numerical experiments have recently been carried out ${ }^{3}$ examining the structure, propagation pathways, and interactions of the North Brazilian current rings with the narrow pathways between islands of the Lesser Antilles. The influence of the Kuroshio current on westward propagating eddies near the Luzon Strait has been modelled using the Princeton Ocean Model. ${ }^{4}$ Laboratory experiments have also been conducted on a single self-propagating vortex near two islands ${ }^{5}$ showing that the interaction depends on the size of the vortex and the distance between the islands.

The abyssal ocean contains many disjointed ridges, punctuated by gaps. A variety of oceanographic processes have been considered in such regions. For example, the flow of the stratified abyssal ocean in the presence of a partially blocked meridional barrier ${ }^{6}$ and the exchange flow between large-scale ocean basins through narrow gaps. ${ }^{7}$ These gap regions have also been modelled as a "porous" barrier. ${ }^{8}$

Idealised models which assume two-dimensional flow of an inviscid fluid have been used to construct the Hamiltonian, or Kirchhoff-Routh path function, ${ }^{9}$ for a single point vortex near a gap, in an infinitely long and infinitesimally thin straight barrier in the rigid lid case (i.e., infinite Rossby 
radius). ${ }^{10}$ The main result is that vortices that start far upstream of the gap at greater than half the gap width from the barrier leap across the gap. Vortices far upstream starting closer to the barrier pass through the gap. Subsequently, vortex motion near barriers with two gaps was studied ${ }^{11}$ and then later for barriers having an arbitrary number of gaps. ${ }^{12}$ More recently ${ }^{13}$ analytical trajectories have been found for a barotropic line vortex near a gap in a wall within a channel with a uniform current. Furthermore, numerical trajectories of finite-area patches were computed and compared to the results found through laboratory experiments conducted within a homogeneous rotating fluid, showing good agreement for vortices that pass through the gap completely, i.e., without splitting. These "ideal" models are mathematically equivalent to single-layer quasi-geostrophic dynamics in the limit of infinite Rossby radius of deformation (i.e., the rigid lid limit). While these exact solutions are interesting and useful, in an oceanographic context it is natural to consider the more realistic case of having a finite Rossby radius of deformation.

The present work aims to generalise the work of Ref. 10 by calculating trajectories of point vortices near gaps at finite Rossby radii. The key non-dimensional parameter is $a$, the ratio of the Rossby radius of deformation and the half-width of the gap. Quasi-geostrophic dynamics in a single layer with reduced gravity is used to derive an integral equation whose solution gives the velocity field at the vortex, enabling its trajectory to be computed. For finite $a$ the integral equation must be solved numerically, unlike the rigid lid case $(a \rightarrow \infty),{ }^{10}$ where complex variable methods give exact point vortex trajectories.

Point vortex trajectories are found for a range of finite Rossby radii using the integral equation formulation. For finite area patches of constant vorticity, the integral equation is combined with contour dynamics. ${ }^{14}$ The trajectories of both point vortex and vortex patch models are analysed and compared.

The effect of background flows on the vortex motion is incorporated by appropriately modifying the integral equations and solving using the same techniques.

\section{FORMULATION}

\section{A. Statement of the problem}

A single layer of reduced gravity fluid in a flat bottomed ocean of depth $H$ is considered. In the quasi-geostrophic limit, the potential vorticity $q$ is conserved where

$$
q=\nabla^{2} \psi-\frac{1}{L_{R}^{2}} \psi .
$$

Here, $\psi$ is the geostrophic streamfunction from which the velocity field can be recovered according to $d \mathbf{x} / d t=\mathbf{k} \times \nabla \psi$. The length scale, $L_{R}=\sqrt{g^{\prime} H} / f$, is the Rossby radius of deformation. Here, $f$ is the constant Coriolis parameter and $g^{\prime}$ is the reduced gravity. The rigid lid limit is $L_{R} \rightarrow \infty$, for which the Eulerian dynamics are recovered.

The vortex is located near a gap in an infinitesimally thin, infinitely long wall. This is equivalent to two semi-infinite barriers on either side of a gap of width $2 \mathrm{~W}$. The task is to compute the trajectory of the vortex with circulation $\kappa$ and determine conditions when the vortex passes through this gap. A schematic of the system is shown in Fig. 1.

With $\psi$ scaled by $|\kappa|$, the potential vorticity $q$ scales like $|\kappa| W^{-2}$. For the horizontal scale $W$ (i.e., half the gap width) is chosen. The non-dimensional potential vorticity $q^{\prime}$ can thus be

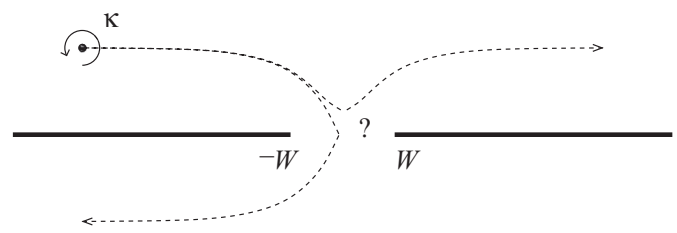

FIG. 1. Possible trajectories of a single vortex of circulation $\kappa$ near a gap in a wall. 
written as

$$
q^{\prime}=\nabla^{2} \psi^{\prime}-\frac{1}{a^{2}} \psi^{\prime},
$$

where $a=\sqrt{g H} / f W$ measures the ratio of the deformation radius $L_{R}$ to the half gap width $W$. Henceforth, the dashes for non-dimensional quantities are omitted.

In an unbounded domain a quasi-geostrophic point vortex of strength $\Gamma$ (where $\Gamma= \pm 1$ in this non-dimensional system), located at $\left(x_{v}, y_{v}\right)$, has streamfunction $\Psi_{P V}$ satisfying

$$
\nabla^{2} \Psi_{P V}-\frac{1}{a^{2}} \Psi_{P V}=\Gamma \delta\left(x-x_{v}\right) \delta\left(y-y_{v}\right)
$$

with solution

$$
\Psi_{P V}\left(x, y, x_{v}, y_{v}\right)=-\frac{\Gamma}{2 \pi} K_{0}\left(\sqrt{\left(x-x_{v}\right)^{2}+\left(y-y_{v}\right)^{2}} / a\right),
$$

where $K_{0}$ is the modified Bessel function of the second kind of order zero. Note that the modified Bessel function structure of the point vortex implies a far-field gradient of the streamfunction (i.e., the velocity induced by the vortex) which decays algebraically for infinite $a$ (i.e., the rigid lid case) but decays exponentially for finite $a$.

In addition to the vortex induced velocity field, there is a vorticity free velocity field, with streamfunction $\psi$, satisfying

$$
\nabla^{2} \psi-\frac{1}{a^{2}} \psi=0
$$

such that the total normal flow at the barrier vanishes. That is, the two half-lines of the barrier are streamlines so that on each barrier

$$
\Psi^{\prime}=\psi+\Psi_{P V}=\text { const. }
$$

The difference in the values of these two constants gives the fixed volume flux through the gap. For simplicity, the analysis is presented first for no flux through the gap so both constants can be taken to be zero and examples with background flux are given later in Sec. III C.

For a barrier running along the $\mathrm{x}$ axis with no gap, the method of images gives

$$
\psi \equiv-\Psi_{P V}\left(x_{v},-y_{v}\right)
$$

and the vortex propagates parallel to the barrier. Clearly this solution is not applicable with a gap present (since it implies the existence of an additional vortex in the flow domain at $\left(x_{v}, y_{v}\right)$ ) and another method must be used to find $\psi$. In the limit $a \rightarrow \infty$, advantage can be taken of the known behaviour of the Hamiltonian under conformal mapping. ${ }^{9}$ By mapping the single gap geometry to a half-space, Johnson and McDonald ${ }^{10}$ were able to obtain an explicit expression for the vortex Hamiltonian in the single-gap geometry. This in turn gives the vortex trajectories explicitly. For a finite $a$, there is no such rule for the behaviour of the Hamiltonian under conformal mapping.

\section{B. Integral equation: Vortex sheet over the barriers}

To find $\psi(x, y)$, it is required to solve the following boundary value problem:

$$
\begin{gathered}
\nabla^{2} \psi-\frac{1}{a^{2}} \psi=0, \\
\psi(x, 0)=-g(x),|x|>1, \\
\psi(r) \rightarrow 0 \text { as } r \rightarrow \infty, \text { where } r=\sqrt{x^{2}+y^{2}},
\end{gathered}
$$

where $g(x)=\Psi_{P V}(x, 0)$ is known.

Let

$$
G\left(x, y, x_{0}, y_{0}\right)=-\frac{1}{2 \pi} K_{0}\left(\sqrt{\left(x-x_{0}\right)^{2}+\left(y-y_{0}\right)^{2}} / a\right)
$$


be the infinite space Green's function for the Helmholtz equation (8). Then,

$$
\nabla^{2} G-\frac{1}{a^{2}} G=\delta\left(x-x_{0}\right) \delta\left(y-y_{0}\right) .
$$

Now, integrating over all space and using Green's Theorem and (10) gives

$$
\iint_{D}\left(\psi \nabla^{2} G-G \nabla^{2} \psi\right) \mathrm{d} x \mathrm{~d} y=\int_{b}\left(\psi \frac{\partial G}{\partial y}-G \frac{\partial \psi}{\partial y}\right) \mathrm{d} x,
$$

where the right hand side is the line integral around the semi-infinite barriers, i.e., extending from $(-\infty,-1]$ and $[1, \infty)$ above the barriers $\left(y=0^{+}\right)$and $(\infty, 1]$ and $[1, \infty)$ below the barriers $\left(y=0^{-}\right)$.

Since $\psi(x)$ is continuous across $y=0$,

$$
\int_{b} \psi(x) \frac{\partial G}{\partial y}\left(x, 0, x_{0}, y_{0}\right) \mathrm{d} x=0 .
$$

Hence, using (8), (12), and (14), (13) can be simplified to give

$$
\psi\left(x_{0}, y_{0}\right)=-\int_{b} \lambda(x) G\left(x, 0, x_{0}, y_{0}\right) \mathrm{d} x
$$

where $\lambda(x)=\partial \psi / \partial y$ on $|x|>1, y=0$.

Differentiation of (15) with respect to $y_{0}$ and $x_{0}$ gives the velocity at $\left(x_{0}, y_{0}\right)$

$$
\begin{gathered}
u\left(x_{0}, y_{0}\right)=\int_{b} \lambda(x) \frac{\partial G}{\partial y_{0}}\left(x, 0, x_{0}, y_{0}\right) \mathrm{d} x, \\
v\left(x_{0}, y_{0}\right)=-\int_{b} \lambda(x) \frac{\partial G}{\partial x_{0}}\left(x, 0, x_{0}, y_{0}\right) \mathrm{d} x .
\end{gathered}
$$

It remains to find $\lambda(x)$. On the barrier (15) gives

$$
\begin{aligned}
g\left(x_{0}\right) & =\int_{b} \lambda(x) G\left(x, 0, x_{0}, 0\right) \mathrm{d} x \\
& =2 \int_{1}^{\infty} \lambda(x) G\left(x, 0, x_{0}, 0\right) \mathrm{d} x+2 \int_{-\infty}^{-1} \lambda(x) G\left(x, 0, x_{0}, 0\right) \mathrm{d} x
\end{aligned}
$$

which, since $g\left(x_{0}\right)$ is known, is an integral equation for the unknown $\lambda(x)$. Once $\lambda(x)$ is determined, (16) and (17) then give the velocity field at any arbitrary point $\left(x_{0}, y_{0}\right)$.

\section{SOLUTION OF THE INTEGRAL EQUATION}

\section{A. Simplification of the integral equation}

The integral equation (18) can be solved by discretising the integrals and solving the subsequent system of linear equations by matrix inversion. Anticipating later consideration of the vortex patch case where the streamfunction along the barriers is not readily computed, but the normal velocity on the barriers, $n(x)$, is using contour dynamics, (18) is differentiated with respect to $x_{0}$, to give

$$
\begin{aligned}
\frac{\partial g}{\partial x_{0}}\left(x_{0}\right) & =n\left(x_{0}\right) \\
& =2 \int_{1}^{\infty} \lambda(x) \frac{\partial G}{\partial x_{0}}\left(x, 0, x_{0}, 0\right) \mathrm{d} x+2 \int_{-\infty}^{-1} \lambda(x) \frac{\partial G}{\partial x_{0}}\left(x, 0, x_{0}, 0\right) \mathrm{d} x,
\end{aligned}
$$

where $n\left(x_{0}\right)$ is the normal velocity along the barrier owing to the vortex alone. 
Now, the normal velocity along the barriers can be split up into even and odd parts,

$$
\begin{aligned}
n\left(x_{0}\right) & =\frac{n\left(x_{0}\right)+n\left(-x_{0}\right)}{2}+\frac{n\left(x_{0}\right)-n\left(-x_{0}\right)}{2} \\
& =n_{e}\left(x_{0}\right)+n_{o}\left(x_{0}\right) .
\end{aligned}
$$

For the even case, let unknown function being sought be $\lambda_{1}(x)$ and for convenience, write

$$
\frac{\partial G}{\partial x_{0}}\left(x, 0, x_{0}, 0\right)=\mathcal{K}_{a}\left(x-x_{0}\right),
$$

and note from (11) $\mathcal{K}_{a}\left(x-x_{0}\right)=-\mathcal{K}_{a}\left(x_{0}-x\right)$.

Hence, from (19),

$$
\begin{aligned}
n_{e}\left(x_{0}\right) & =\int_{-\infty}^{-1} \lambda_{1}(x) \mathcal{K}_{a}\left(x-x_{0}\right) \mathrm{d} x+\int_{1}^{\infty} \lambda_{1}(x) \mathcal{K}_{a}\left(x-x_{0}\right) \mathrm{d} x \\
& +\int_{-\infty}^{-1} \lambda_{1}(x) \mathcal{K}_{a}\left(x+x_{0}\right) \mathrm{d} x+\int_{1}^{\infty} \lambda_{1}(x) \mathcal{K}_{a}\left(x+x_{0}\right) \mathrm{d} x,
\end{aligned}
$$

from which it follows that $\lambda_{1}(x)$ is odd and the integral equation (19) can be re-written so that the integration is along one barrier, i.e.,

$$
\begin{aligned}
n_{e}\left(x_{0}\right) & =2 \int_{1}^{\infty} \lambda_{1}(x) \mathcal{K}_{a}\left(x+x_{0}\right) \mathrm{d} x+2 \int_{1}^{\infty} \lambda_{1}(x) \mathcal{K}_{a}\left(x-x_{0}\right) \mathrm{d} x, \\
& =2 \int_{1}^{\infty} \lambda_{1}(x)\left(\mathcal{K}_{a}\left(x-x_{0}\right)+\mathcal{K}_{a}\left(x+x_{0}\right)\right) \mathrm{d} x .
\end{aligned}
$$

Similarly, for the odd case where letting the unknown function being sought be $\lambda_{2}(x)$, it follows:

$$
n_{o}\left(x_{0}\right)=2 \int_{1}^{\infty} \lambda_{2}(x)\left(\mathcal{K}_{a}\left(x-x_{0}\right)-\mathcal{K}_{a}\left(x+x_{0}\right)\right) \mathrm{d} x .
$$

\section{B. Numerical solution of the integral equation}

Integral equations (23) and (24) are singular since for $x_{0}>0$,

$$
\mathcal{K}_{a}\left(x-x_{0}\right)=\frac{A}{x-x_{0}}+\mathrm{O}(1), \text { as } x \rightarrow x_{0},
$$

where $A$ is a constant. Thus, the integrals in (23) and (24) need to be interpreted in a Cauchy principal value sense. The solutions $\lambda_{1}(x)$ and $\lambda_{2}(x)$ are also expected to have inverse square root singularities at the barrier tip. That is,

$$
\lambda_{1,2}(x) \sim \frac{B}{\sqrt{x-1}} \text { as } x \rightarrow 1,
$$

where $B$ is a constant. This is because for small distances from the barrier tip the solution of the Helmholtz equation should give a velocity field which tends to that governed by Laplace's equation. It is well known that such a velocity field has an inverse square root singularity.

Let

$$
\mathcal{E}\left(x, x_{0}\right)=\mathcal{K}_{a}\left(x-x_{0}\right)+\mathcal{K}_{a}\left(x+x_{0}\right),
$$

and approximate (23) as

$$
n_{e}\left(x_{0}\right)=2 \int_{1}^{1+\epsilon} \lambda_{1}(x) \mathcal{E}\left(x, x_{0}\right) \mathrm{d} x+2 \int_{1+\epsilon}^{L} \lambda_{1}(x) \mathcal{E}\left(x, x_{0}\right) \mathrm{d} x,
$$

where $L \gg 1$ is the truncation length of the barrier and $0<\epsilon \ll 1$. The first integral in (28) encompasses the inverse square root singularity of $\lambda_{1}(x)$ and, provided $x_{0}$ is not close to $x=1$ (i.e., the vortex is not near the barrier tip), is integrable and behaves like $\sqrt{\epsilon}$ as $\epsilon \rightarrow 0$. Thus, to a good 
approximation, we take $\epsilon=0$ and (28) becomes

$$
n_{e}\left(x_{0}\right) \simeq 2 \int_{1}^{L} \lambda_{1}(x) \mathcal{E}\left(x, x_{0}\right) \mathrm{d} x .
$$

The integral in (29) is discretised into $N$ points,

$$
x_{i}=1+(i-1) h, i=1, \ldots, N,
$$

where $h=(L-1) /(N-1)$. Let

$$
x_{0}^{j}=x_{j}+\frac{h}{2}, j=1, \ldots, N,
$$

be the mid-points of the $\left[x_{i}, x_{i+1}\right]$ intervals values along the barrier. Note that the final mid-point $j$ $=N$ lies outside $[1, L]$. This is fine, since the choice of truncation length is arbitrary; and data for $n_{e}\left(x_{0}^{N}\right)$ are available there. It can be shown ${ }^{15}$ that, using the trapezoidal rule,

$$
\int_{1}^{L} \mathcal{E}\left(x, x_{0}\right) \lambda_{1}(x) \mathrm{d} x \simeq \sum_{i=1}^{N} \lambda_{1}\left(x_{i}\right) \mathcal{E}\left(x_{i}, x_{0}^{j}\right) w_{i},
$$

where $w_{i}$ are the usual trapezoidal weights

$$
w_{i}= \begin{cases}\frac{h}{2} & i=1, N \\ h & \text { otherwise. }\end{cases}
$$

That is, by considering $x_{0}^{j}$ as the mid-points, the presence of the $\left(x-x_{0}^{j}\right)$ singularity is accounted for.

Hence, the integral equation (23) can be approximated by the $N \times N$ linear system,

$$
\sum_{i=1}^{N} \mathcal{E}\left(x_{i}, x_{0}^{j}\right) \lambda_{1}\left(x_{i}\right) w_{i} \simeq n_{e}\left(x_{0}^{j}\right), j=1, \ldots, N,
$$

where the unknowns $\lambda_{1}\left(x_{i}\right)$ are found by numerically inverting the coefficient matrix. Similarly, the solution $\lambda_{2}(x)$ to $(24)$ can be found.

Once the $\lambda_{1,2}\left(x_{i}\right), i=1, \ldots, N$, are determined, the velocity field at any point $\left(x_{0}, y_{0}\right)$ is found by truncating and numerically evaluating the integrals in (16) and (17), again using the trapezoidal rule.

\section{Background flows}

The advection of vortices by ambient currents and background flows will influence their trajectories. Two background flows are considered here.

For flow symmetric in $x$ through the gap with streamfunction $\psi_{s}$, we can derive an integral equation identical to (15) with the streamfunction along the boundary given by

$$
\psi_{s}(x, 0)=\frac{\mathcal{F}}{2 \pi} \operatorname{sgn}(x),|x|>1,
$$

where $\mathcal{F}$ is the flux through the gap. Exploiting the fact that $\psi(x, 0)$ is odd yields the following integral equation:

$$
\frac{\mathcal{F}}{2 \pi}=2 \int_{1}^{\infty} \lambda_{s}(x)\left(G\left(x, 0, x_{0}, 0\right)-G\left(x, 0,-x_{0}, 0\right)\right) \mathrm{d} x,
$$

which can be solved as before for the unknown $\lambda_{s}(x)$. Once $\lambda_{s}(x)$ is known then the streamfunction at any point $\left(x_{0}, y_{0}\right)$ is found from

$$
\psi_{s}\left(x_{0}, y_{0}\right)=-\int_{b} \lambda_{s}(x) G\left(x, 0, x_{0}, y_{0}\right) \mathrm{d} x .
$$

Another non-trivial flow can be found by letting $\psi_{a}(x, 0)=\mathcal{F} / 2 \pi,|x|>1$, and insisting $\psi_{a} \rightarrow 0$ as $r \rightarrow \infty$. This corresponds to a non-trivial flow with zero net flux through the gap, 


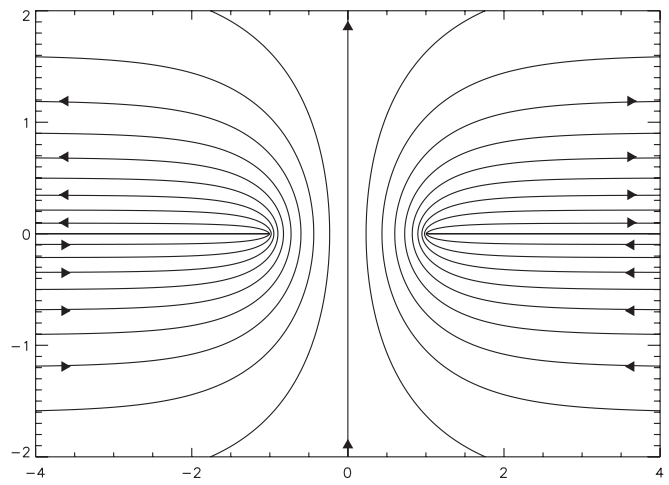

(a)

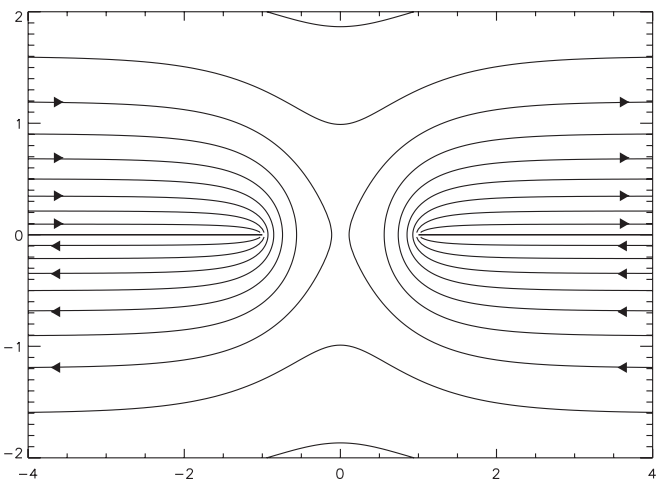

(b)

FIG. 2. (a) Streamlines for the symmetric flow through the gap with $\mathcal{F}=1$ and contour values of $\psi_{s}(x, y)=n / 10$ for $n=1$, ..., 9. (b) Streamlines for the anti-symmetric flow through the gap with $\mathcal{F}=1$ and contour values of $\psi_{a}(x, y)=n / 10$ for $n$ $=1, \ldots, 9 . a=1$ in both (a) and (b). The arrow-heads on the streamlines indicate the direction of flow.

such that the through gap velocity is anti-symmetric. Such a flow field occurs near a gap when one of the basins connected by the gap is closed. By mass conservation there is zero net flux across the gap as in Fig. 2(b). Further, owing to the linearity of the governing equation, any linear combination of the flow fields shown in Figs. 2(a) and 2(b) generates all possible background flow fields that have bounded speed at infinity. This results in the following integral equation, for $\lambda_{a}(x)=\partial \psi_{a} / \partial y$ on $|x|>1, y=0$,

$$
\frac{\mathcal{F}}{2 \pi}=2 \int_{1}^{\infty} \lambda_{a}(x)\left(G\left(x, 0, x_{0}, 0\right)+G\left(x, 0,-x_{0}, 0\right)\right) \mathrm{d} x .
$$

Integral equations (36) and (38) are solved using the procedure described in Sec. III B and the resulting streamlines with $\mathcal{F}=1$, for symmetric flow, $\psi_{s}$ and anti-symmetric flow, $\psi_{a}$ are shown in Fig. 2. Note that the anti-symmetric case shown in Fig. 2(b) has no analogy in the rigid-lid case: as $a \rightarrow \infty, \psi_{a} \rightarrow \mathcal{F} / 2 \pi$ everywhere and the flow becomes stagnant. In principle, any linear combination of $\psi_{a}$ and $\psi_{s}$ can be used to generate a non-trivial background flow.

\section{RESULTS}

\section{A. Point vortex motion}

The method described in Sec. III C to find the velocity field owing to the presence of the barrier enables the motion of point vortices and finite area patches to be computed. A large number of points, $N=2^{11}$, was used to discretise the barrier on the interval $1 \leq x \leq L$, where $L=13 a$. The truncation length $13 a$ being sufficiently large so that the typical magnitude of the normal velocity induced by the vortices with $\left|x_{v}\right| \leq 4$ on the barrier was of order $10^{-6}$ at $x=L$.

Once $\lambda(x)$ is computed, the velocity at the vortex is found using (16) and (17) and is advected using the fourth order Runge-Kutta routine with a time-step of at most $\Delta t=0.1$.

An alternative numerical method using the rigid lid solution as the basis of an iterative solution of the integral equation which is valid for $a>1$ was used to verify the results of the matrix method for a range of $a>1$. The details of this method can be found in the Appendix.

When in the far field, the point vortex follows the rigid lid trajectory regardless of $a$ as the presence of the gap is not felt, namely, the vortex behaves as it were near an infinitely long wall and propagates parallel to it. Although the trajectories are the same, the vortex propagation velocities are different with a decrease in the velocity as $a$ is decreased.

When sufficiently close to the barrier, for $a=10$, the presence of the gap is eventually felt and the vortex starts dipping towards the gap and eventually passes through the gap, with a trajectory resembling the motion of a vortex near a semi-infinite barrier, as the effect of the second barrier cannot be felt. When starting further from the barrier the downstream barrier influences the motion 


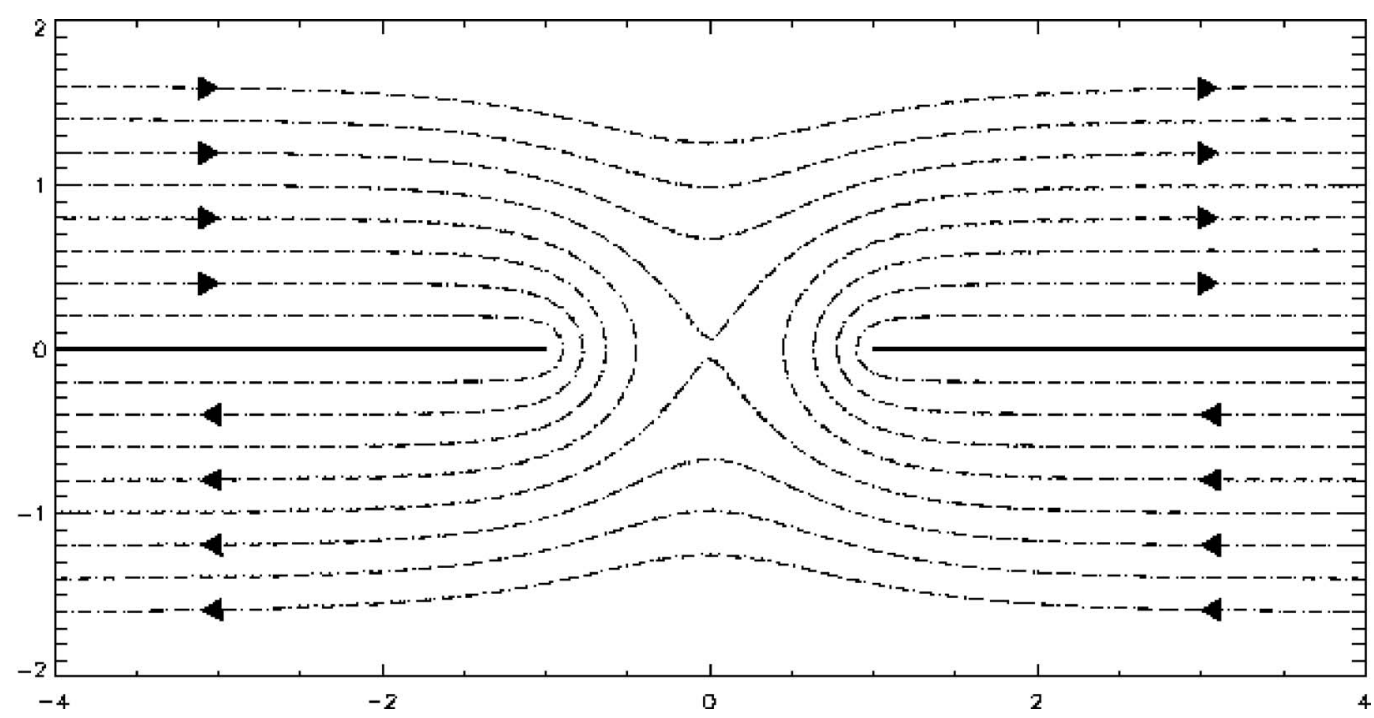

FIG. 3. A comparison between the analytical vortex $(\Gamma=1)$ trajectories for the rigid lid case, $a \rightarrow \infty$ (dashed lines) and the numerically calculated trajectories for $a=10$ (dotted lines) with zero background flow. The numerical trajectories are for individual vortices starting at $\left(x_{v}, y_{v}\right)=( \pm 4, \mp 0.2 n), n=1, \ldots, 8$. The arrow-heads on the trajectories indicate the direction of vortex motion.

before the vortex can pass through the gap and the vortex leaps across the gap. A separatrix divides the two behaviours such that critical distance from the barrier far upstream of the gap is close to unity. Trajectories are shown in Fig. 3. As expected, for this large value of $a$ the behaviour of the point vortex is essentially that of the rigid lid case studied by Johnson and McDonald ${ }^{10}$ (see also Fig. 3), who showed that the critical trajectory on the separatrix is such that $|y|=1$ as $x \rightarrow \infty$.

Figure 4 compares vortex trajectories for $a=1$ and the $a \rightarrow \infty$ (exact) case. An immediate feature is that when $a=1$ there is an enhanced capability for vortices to pass through the gap. For example, the $a=1$ trajectory starting at $(-4,1)$ passes through the gap whereas it lies just outside the separatrix for $a=\infty$. This is because it takes longer for the vortex to feel the effect of the gap owing to the more rapid (exponential) decay of the velocity field of the $a=1$ vortex compared to the slower algebraic decay as $a \rightarrow \infty$. Once it is above the gap, the effect of the downstream barrier

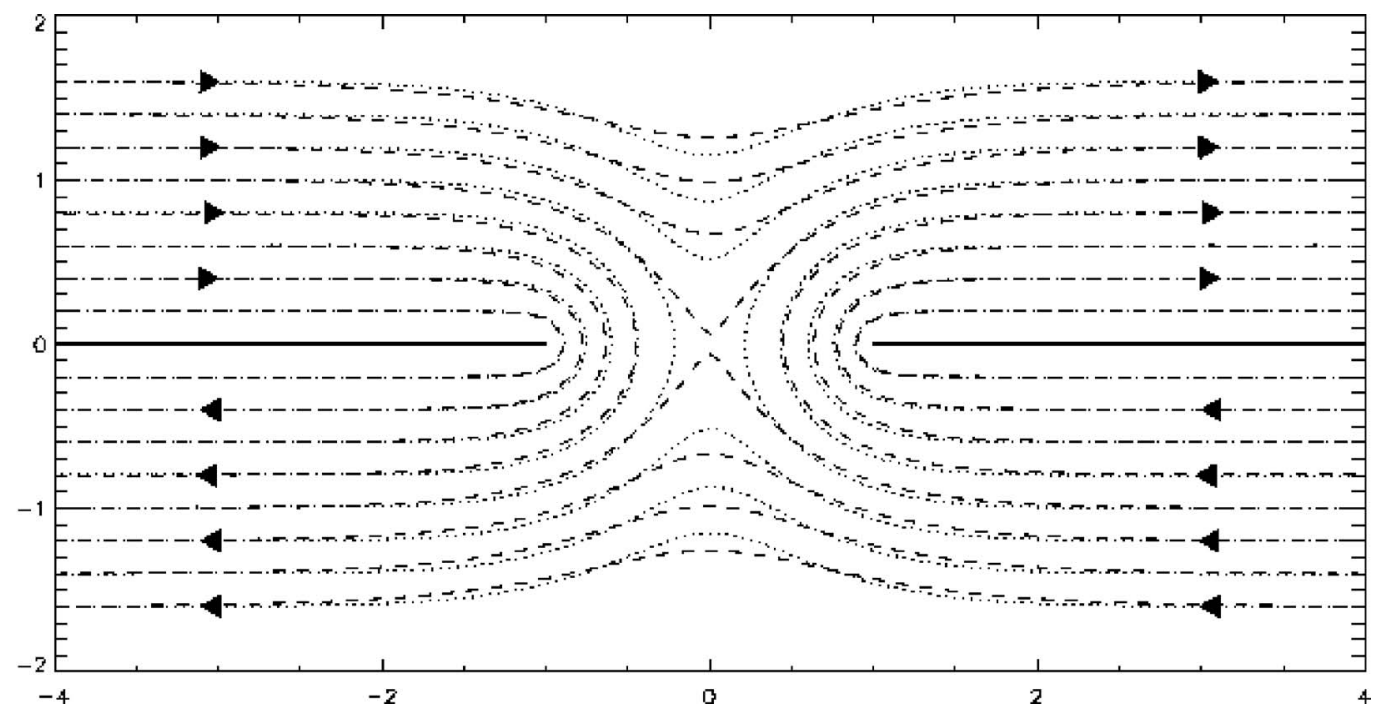

FIG. 4. Same as in Fig. 3 but with $a=1$ (dotted lines). 


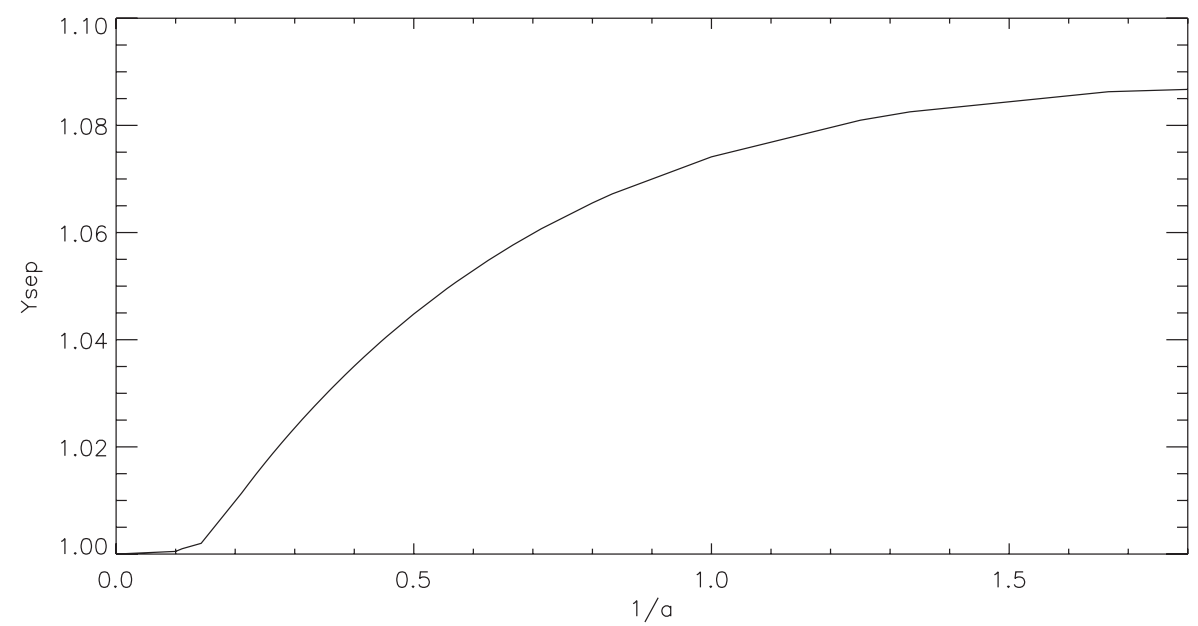

FIG. 5. Value of $y_{\text {sep }}$ against $1 / a$. The tendency for vortices to pass through the gap is increased with decreasing $a$.

is felt less strongly than for the rigid lid case and therefore, it does not leap across, but it passes through.

The numerical method can be used to quantify the effect the Rossby radii has on the separatrix. A point vortex located at $\left(10^{-6}, 10^{-6}\right)$ can be advected until the vortex passes the line, $|x|=5$, where the motion becomes effectively that of a point vortex near a wall. Let this distance $y$ be denoted as $y_{s e p}(1 / a)$. For the rigid lid case, $y_{s e p}(0)=1$. This function has been computed for various values of $a$ and plotted as a function of 1/a in Fig. 5 which clearly shows that $y_{s e p}$ is further from the $\mathrm{x}$ axis as $a$ decreases, i.e., $a^{-1}$ increases. The effect is relatively minor, there being less than $10 \%$ change in $y_{\text {sep }}$ over the range of $a$ tested.

Figure 6 shows point vortex trajectories with symmetric flow through the gap of unit strength $\mathcal{F}=1$, with $a=1$. For $x>0, y<0$, the velocity induced on the vortex owing to the background flow and its self-advection along the wall are in the same direction. Hence, vortices starting further from the wall will pass through the gap. For $x<0, y>0$, the background and vortex self-advection velocities are in opposite directions, hence, vortices are pushed back and need to be very close to the wall in order to generate sufficient speed from self-advection to overcome the background flow.

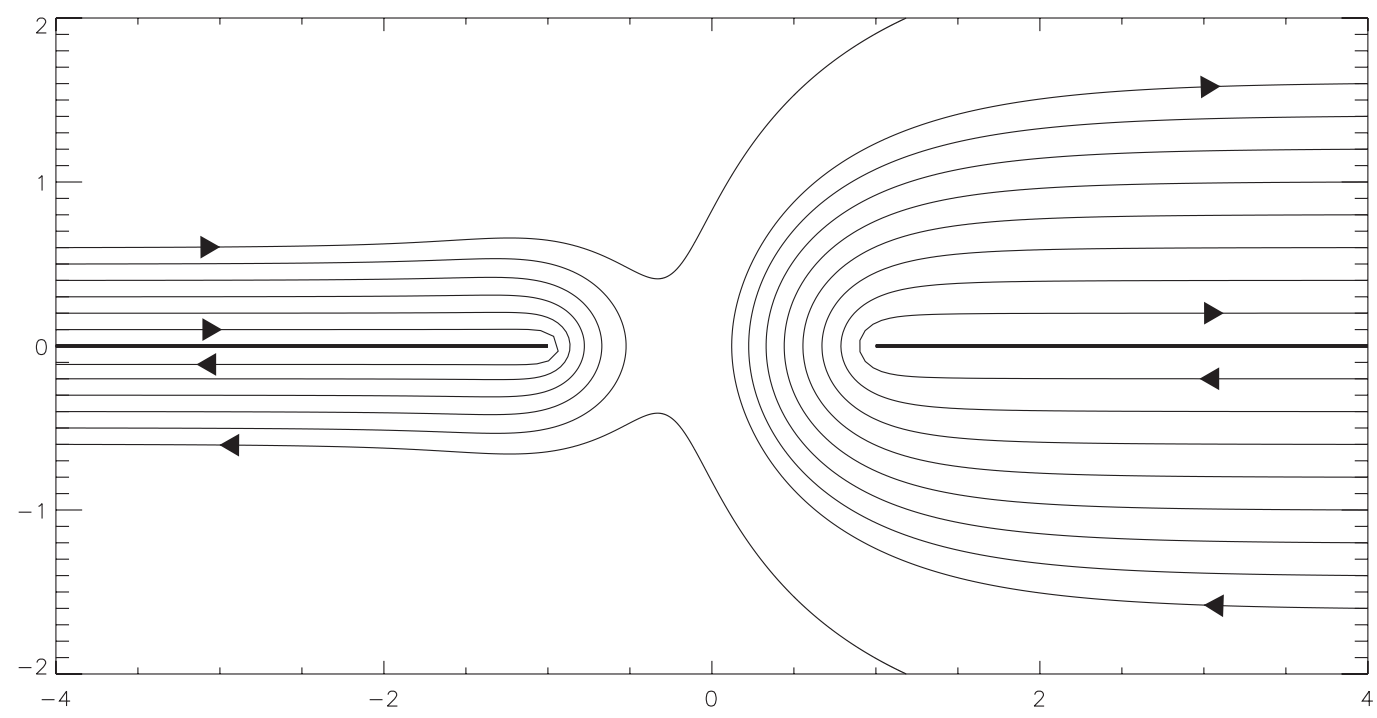

FIG. 6. The effect of the symmetric flow (as in Fig. 2(a)) of unit strength, $\mathcal{F}=1$, on the trajectories of single point vortices $(\Gamma=1)$ starting from $(-4, .1 n), n=1, \ldots, 7$ and $(4, .2 n), n=1, \ldots, 7$ with $a=1$. 


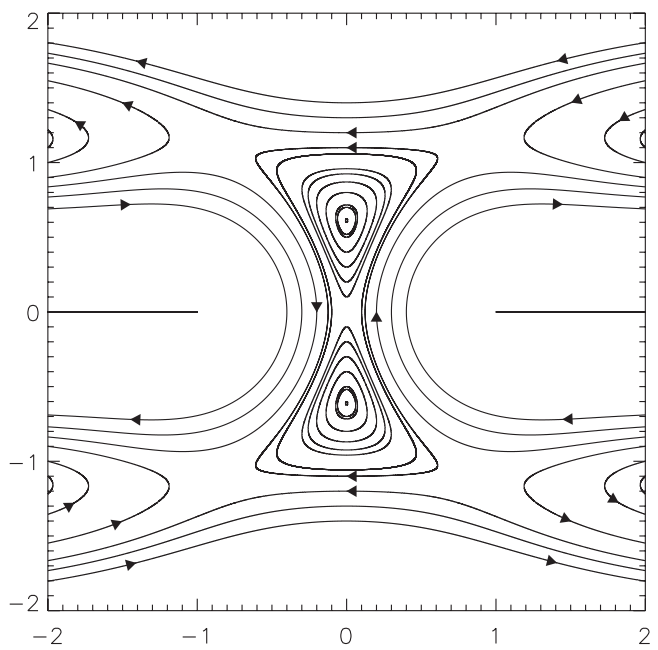

(a)

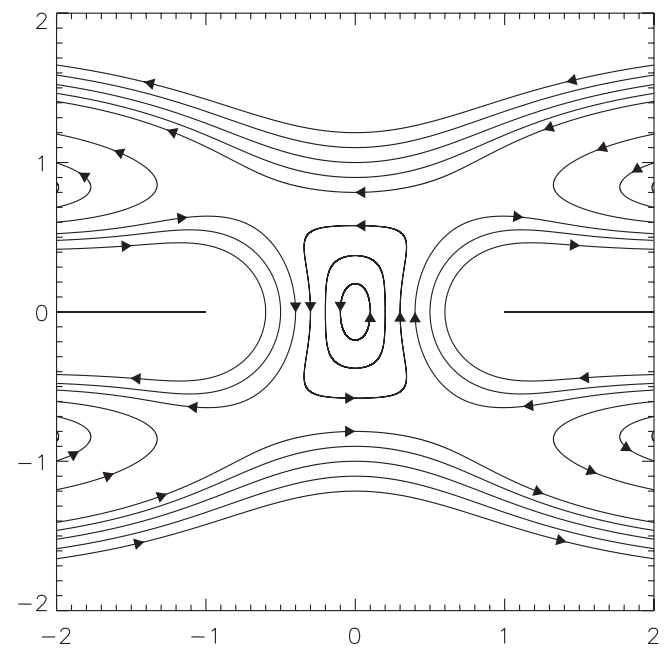

(b)

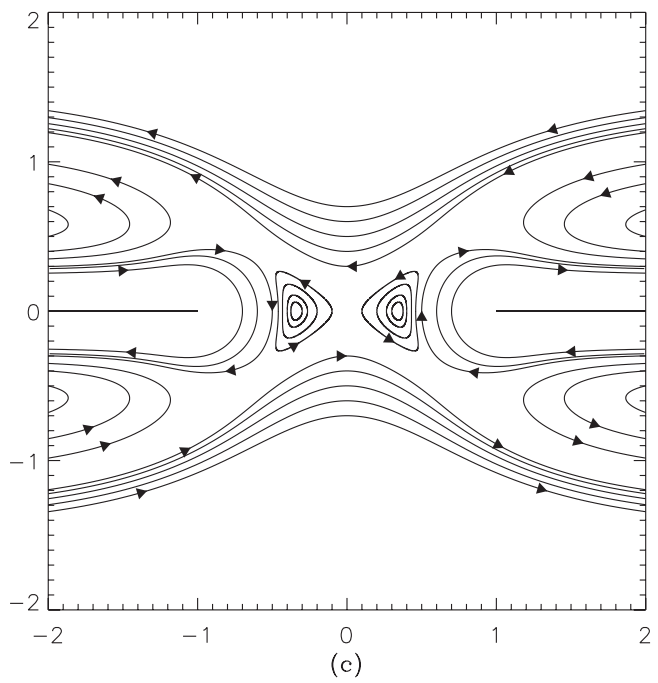

FIG. 7. Vortex trajectories $(\Gamma=1)$ over a range of anti-symmetric flows with $a=1$. The strength of the flows in each figure is $-\mathcal{F}=0.6,1,1.6$ in (a), (b), and (c), respectively. The direction of the background flow is the same as in the streamlines in Fig. 2(b).

In the presence of anti-symmetric background flow, there exist a variety of vortex behaviours depending on the flow strength $\mathcal{F}$. For example, there exist closed trajectories where vortices remain locally trapped (see Fig. 7). For $\mathcal{F}=-0.6$ (Fig. 7(a)), there are four primary regions within the flow including the hour glass shape which contains a region of trapped vortex paths. There are also three stagnation points on the $y$ axis including one which is unstable at the origin. For large $|x|$, some vortices approach the gap but are turned back, travelling back towards their initial position owing to the background flow. Note that vortices sufficiently far from the barrier also propagate against the direction of self-advection owing to the background flow.

Increasing the strength of the background flow squashes the hour glass so that its height ( $y$-direction) decreases and its width ( $x$-direction) increases as the two stable (elliptic) stagnation points on the y axis coalesce. In Fig. 7(b) $(\mathcal{F}=-1)$, the hour glass contains closed paths (with their semi-major axes lying on the y axis). Interestingly, the centre of the gap has gone from an unstable hyperbolic point Fig. 7(a) to an elliptic point Fig. 7(b). In Fig. 7(c), the strongest background flow $(\mathcal{F}=-1.6)$, the hour glass is further squashed and the stagnation points are pushed further out 


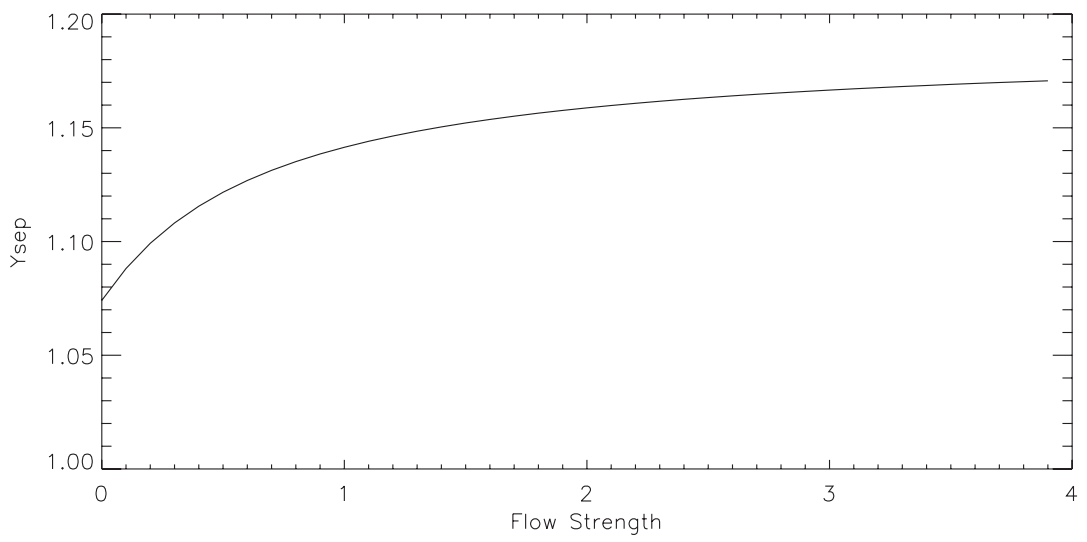

FIG. 8. Value of $y_{\text {sep }}$ as a function of the strength $\mathcal{F}$ of anti-symmetric flow for $a=1$.

towards the tips of the barriers. This behaviour suggests the possibility of trapped eddies near bay exits.

Figure 8 shows that for vortices starting $x \rightarrow-\infty, y>0, y_{s e p}$ increases with an increase in anti-symmetric flow strength.

\section{VORTEX PATCH MOTION}

Contour dynamics ${ }^{14}$ enables the motion of a finite area patch of vorticity to be calculated efficiently by advecting the boundary of the vortex patch. The contour dynamics algorithm, in conjunction with contour surgery ${ }^{14}$ which allows for the breaking and joining of contours, is modified to include the gap geometry.

The normal velocity along the barriers owing to a piece-wise constant distribution of vorticity is calculated using the contour dynamics algorithm. As in the case for the point vortices, the normal velocity is split into even and odd parts. The barrier discretised over the interval $1 \leq x \leq 13 a$. The resulting even and odd velocity fields along the barrier are used to compute, by solving the resultant integral equations, the respective streamfunction derivatives $\left(\lambda_{1,2}(x)\right)$ using the same method outlined for the point vortex case. Once obtained, the velocity owing to the barriers is calculated at each node along the contour. This velocity, along with the self-induced patch velocity (computed in a "standard" way by contour dynamics), is then used to advect the nodes on the vortex patch boundary using the fourth order Runge-Kutta routine with $\Delta t=0.1$.

\section{A. Vortex patch normalisation}

This section derives a new way of normalising finite area vortex patches so that the motion at different Rossby radii, patches with the same vorticity but with different sizes and areas (including point vortices) can be compared.

The general solution of the Helmholtz equation (8) with arbitrary boundary conditions around the circle, $r=R$ (which later is taken to be the patch radius) can be written for $r<R$ as

$$
\psi=\sum_{n=0}^{\infty}\left(\alpha_{n} \cos n \theta+\beta_{n} \sin n \theta\right) I_{n}(r / a) .
$$

The area-average value of $\psi$ inside a circular vortex patch is, using (39), proportional to $\alpha_{0}$, the value of $\psi$ at the centre of the patch,

$$
\langle\psi\rangle=\frac{1}{\pi R^{2}} \int_{0}^{R} \int_{0}^{2 \pi} \psi r \mathrm{~d} r \mathrm{~d} \theta=2 \frac{\alpha_{0}}{R^{2}} \int_{0}^{R} I_{0}(r / a) r \mathrm{~d} r=\alpha_{0} \mathcal{S},
$$


where

$$
\mathcal{S}=\frac{2 a}{R} I_{1}(R / a),
$$

with $\mathcal{S}$ increasing monotonically from unity in the rigid lid limit $(a \rightarrow \infty)$ with increasing $R / a$.

The $x$ coordinate of the potential vorticity centroid of a finite area patch can be written for a nonconstant potential vorticity $q(x, y)$ as $x_{c}=\iint x q / \mathcal{Q}$ where $\mathcal{Q}=\iint q$ is the total potential vorticity inside the vortex patch, and all integrals are evaluated over the area of the patch. Conservation of potential vorticity $q$ shows that $\mathcal{Q}$ is a constant of the motion and hence,

$$
\mathcal{Q} \dot{x}_{c}=\iint x \dot{q}=-\iint x \cdot \nabla(\mathbf{u} q)=\iint q \mathbf{u} \cdot \nabla x-\nabla \cdot(x \mathbf{u} q) .
$$

The divergence term is reduced to a line integral around the boundary of the patch, which for a finite patch, vanishes. The first term remains which is just the area integral of the $x$ component of the velocity, $u$, multiplied by $q$. This is written as $u=u_{v}+u_{e}$ where $u_{v}$ is the irrotational velocity induced on the patch in isolation from the infinite domain Green's function and $u_{e}$ is the rotational velocity induced from external sources such as images in boundaries or superposed irrotational background flows. The usual manipulations of the conservation of impulse show ${ }^{9}$ that $u_{v}$ makes no contribution to the integral and therefore,

$$
\dot{x}_{c}=\iint u_{e} q / \mathcal{Q}
$$

which, for a patch of constant potential vorticity $q_{0}$, becomes

$$
\dot{x}_{c}=\left\langle u_{e}\right\rangle,
$$

that is, the simple patch-area average velocity of $u_{e}$. As $u_{e}$ is the derivative of $\psi$ and so satisfies Helmholtz's equation over the patch, by (40) we have

$$
\dot{x}_{c}=\mathcal{S} u_{e}\left(x_{c}, y_{c}\right) \text {. }
$$

The analogous results hold for the $y$ coordinate of the centroid and thus the velocity of the patch centroid is $\mathcal{S}\left(u_{e}, v_{e}\right)=\mathcal{S} \mathbf{u}_{e}\left(x_{c}, y_{c}\right)$, i.e., the same velocity field as felt by a point vortex at $\left(x_{c}, y_{c}\right)$ but multiplied by to the factor $\mathcal{S}$.

The velocity $\mathbf{u}_{\mathbf{e}}$ is still required. For a uniform potential vorticity, $q_{0}$, inside the vortex patch the jump in vorticity is

$$
\nabla^{2} \psi-\frac{1}{a^{2}} \psi= \begin{cases}q_{0} & r<R \\ 0 & r>R\end{cases}
$$

Equation (46) has solution, with $\psi$ constant and having continuous derivative along the patch boundary,

$$
\psi / q_{0}= \begin{cases}-a^{2}-a R K_{0}^{\prime}(R / a) I_{0}(r / a) & r<R, \\ -a R I_{0}^{\prime}(R / a) K_{0}(r / a) & r>R .\end{cases}
$$

The field outside the patch $(r>R)$ can be written as

$$
\psi=\mathcal{S}(\mathcal{Q} / 2 \pi) K_{0}(r / a) .
$$

Recall, (45), that the external field plus any imposed external field then induces a velocity field on a patch that scales with $\mathcal{S}$ so that the patch moves with velocity $\mathcal{S} \mathbf{u}_{e}\left(x_{c}, y_{c}\right)$. Hence, a point vortex can be made to move at the same speed near, say, a boundary as a circular patch of arbitrary radius $R$ provided the circulation of the point vortex $\Gamma_{n}=S^{2} \mathcal{Q}$. As the rigid lid approximation is approached, $a \rightarrow \infty, \Gamma_{n} \rightarrow \mathcal{Q}$, hence, scaling directly with the circulation of the vortex. It is important to note that this "usual" scaling with the vortex circulation only holds in the $a \rightarrow \infty$ limit, and that, more generally, the patch circulation must be scaled by $\mathcal{S}^{2} \mathcal{Q}$.

Figure 9 shows results for the motion of individual patches with different areas moving near a gap in the absence of a background flow field with $a=10$. As can be seen in Fig. 9(a), with 


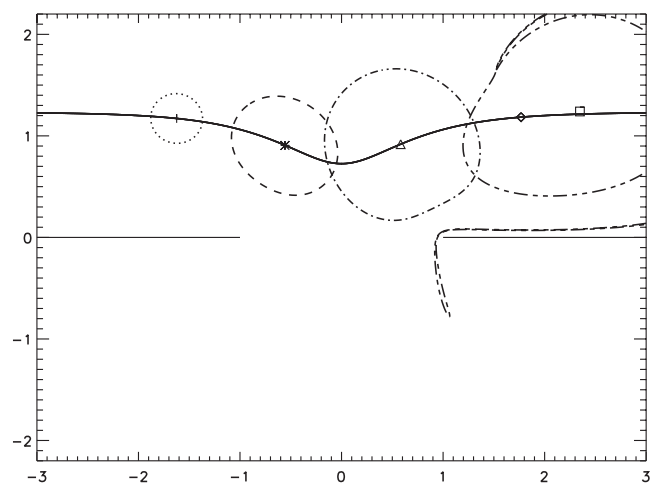

(a)

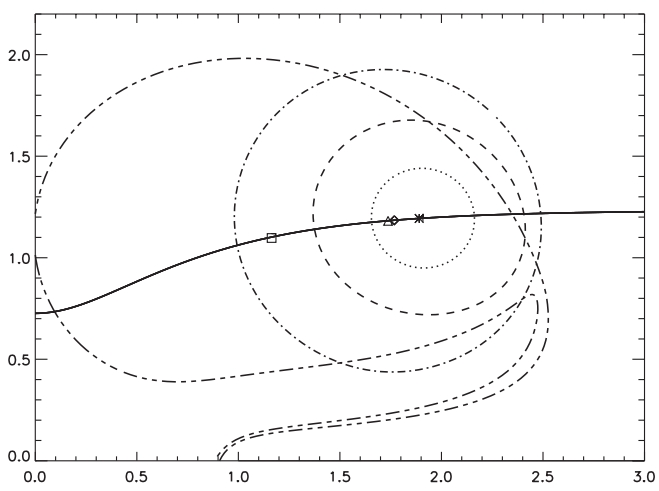

(b)

FIG. 9. The effect of various vortex patch normalisations. (a) Snapshot at $t=28.8$ for the motion of a point vortex $(\diamond)$ and circular vortex patches of radius, $R=0.25,0.5,0.75,1$ with centroids denoted by $(+, *, \triangle, \square)$, respectively. Here, $a=10$, $q=1$ and patch centroids are initially located at $(-2,1.2)$. Circulation of the point vortex is $\Gamma=1$. The solid line indicates the trajectory of a point vortex. (b) same as in (a) but zoomed in to focus on the centroids and with $q$ scaled by the patch size so that $\Gamma=q \pi R^{2}=1$ for all patches.

non-normalised flows with $q=1$, the larger patches travel faster than smaller patches. Note that patches of area $\pi R^{2}<1$ with $R=0.25$ and $R=0.5$ travel slower than a point vortex of strength $\Gamma$ $=1$ whereas patches with area $\pi R^{2}>1$ with $R=0.75$ and $R=1$ travel faster. The largest patch deforms significantly due to the presence of the downstream barrier and begins to shed vorticity causing its centroid to deviate from the equivalent point vortex trajectory. For $a=10$, the patches velocity can be normalised with the circulation of the patches, as seen in Fig. 9(b). The largest patch does slow down considerably due to its interaction with the barrier causing it to become non-circular.

For $a=1$, using the "usual" circulation based scaling, shows that the deviation of the patch speed from the point vortex speed is significant (see Fig. 10(a)). In particular, the deviation is not systematic with $R=0.75$, having the greatest translation speed. Instead, Fig 10(b) has its vorticity $q$ scaled such that $\mathcal{S}^{2} \mathcal{Q}=1$ for all patches. This new scaling shows that there is a systematic deviation from the point vortex speed, i.e., the smaller the radius of the patch, the closer the speed to the point vortex. For patches which remain close to circular throughout their evolution, there is very good agreement in both their trajectories and speed.

Similarly, the effect of background flows can be scaled so that vortex patches of varying size follow the point vortex trajectories with the same speed. In Fig. 11, the symmetric flux through the

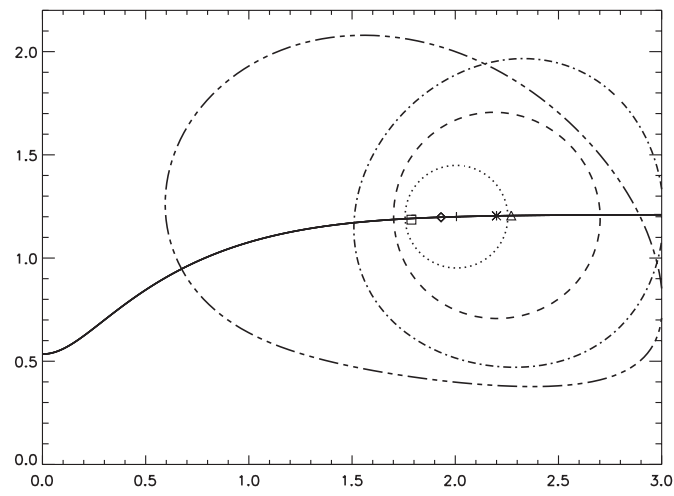

(a)

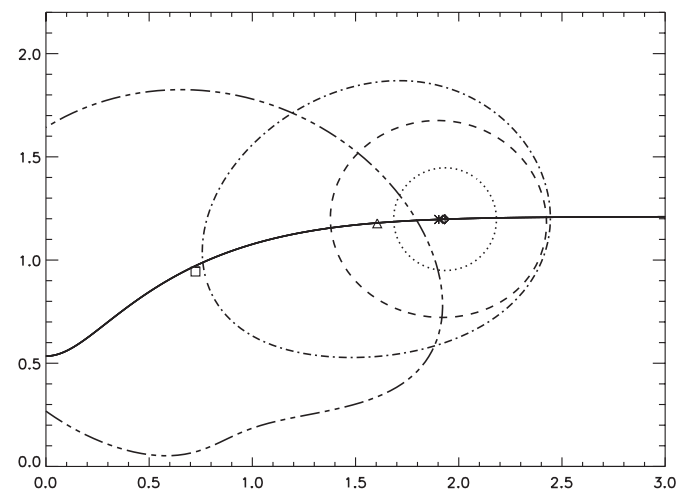

(b)

FIG. 10. (a) Same as in Fig. 9(b) but with $a=1$ and snapshot taken at $t=350$. Note the non-systematic spacing of the patch centroids. (b) Same as in (a) but with vorticity, $q$, scaled so that $S^{2} \mathcal{Q}=1$. Here, vortices which remain close to circular travel at the same speed as a point vortex with strength $\Gamma=1$. 


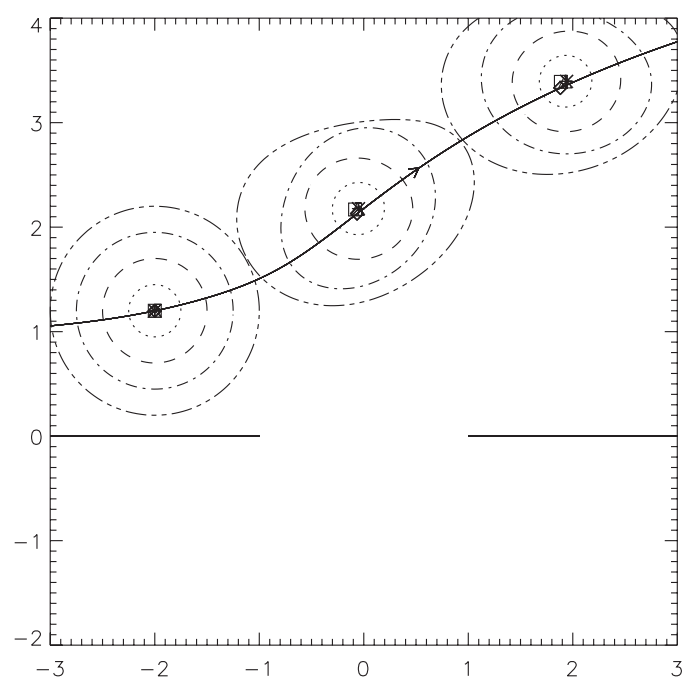

FIG. 11. Motion of patches of varying size with normalised symmetric flow through the gap (as in Fig. 2(a)) with $\mathcal{F}=1 / \mathcal{S}$. Initially, the centroid location of the normalised patches and the point vortex is $(-2,1)$, with the centroids denoted in the same way as in Fig. 9. Snapshots taken at $t=0,61.6,140$ and $a=1$. Centroids are indicated by $(+, *, \Delta, \square)$ symbols.

gap advects the vortex patches in the positive $y$ direction. Here, $a=1$ and the background flow has been scaled appropriately by the factor $\mathcal{S}^{-1}$.

For a positive vorticity patch with the flux reversed, the background flow forces the patch closer to the gap, see Fig. 12. Owing to the significant deformation of the larger patches there is some observable, but small, change in patch speed in comparison to that of a point vortex.

Unlike for a point vortex, the anti-symmetric flux through the gap can result in large deformation of the patch. For example, Fig. 13 shows a vortex patch approaching the centre of the gap where the background flow is strongly sheared causing the vortex to split.

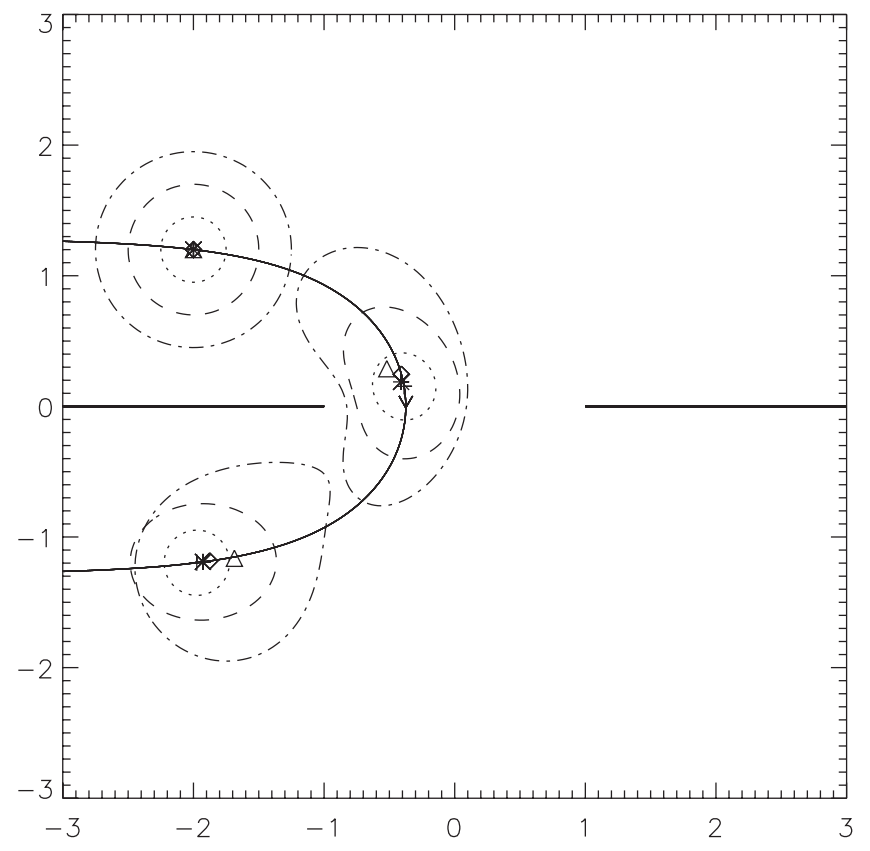

FIG. 12. Same as Fig. 11 but with $\mathcal{F}=-1 / \mathcal{S}$ and $a=1$. Snapshots taken at $t=0,40.8,80$. 

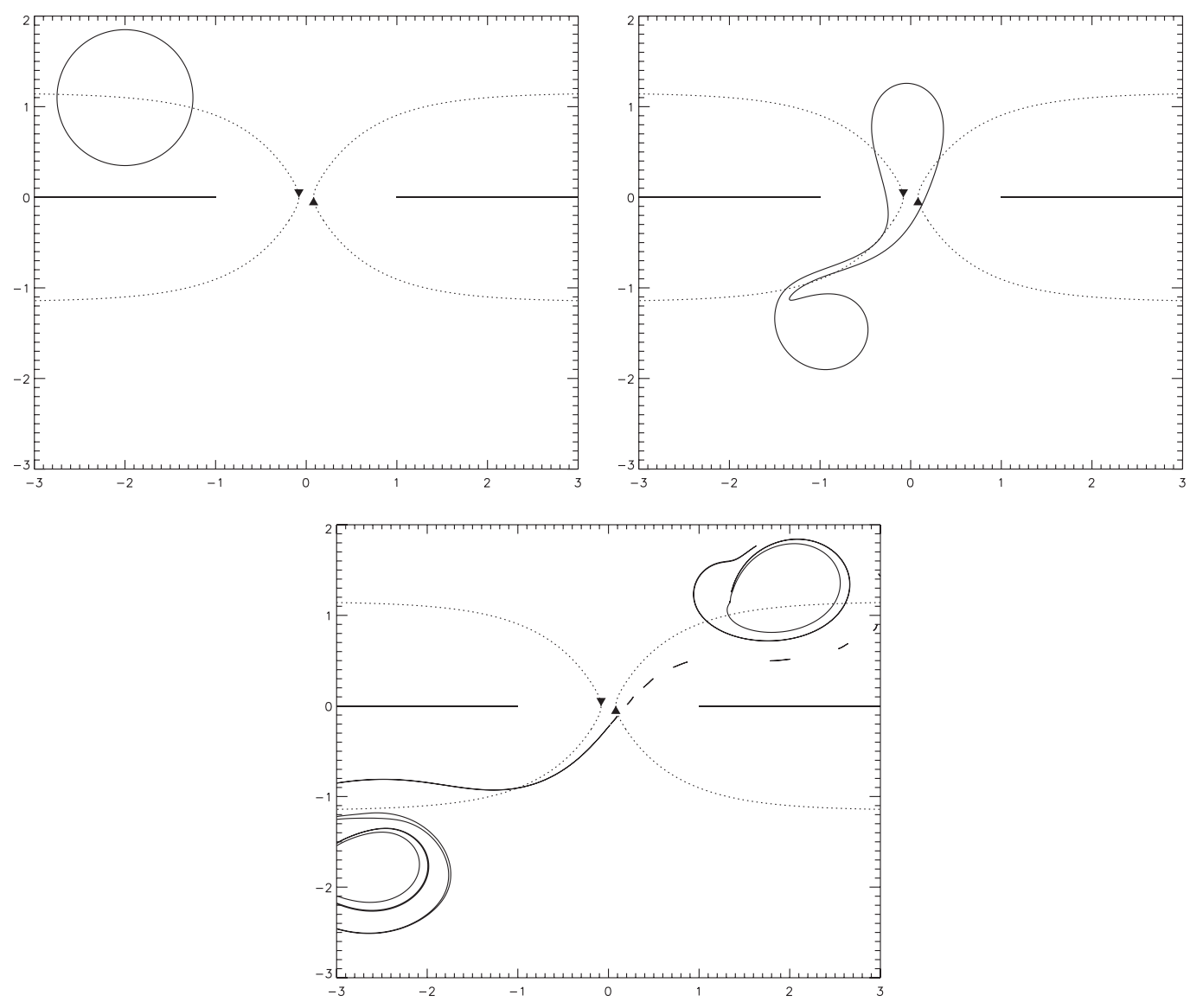

FIG. 13. Motion of a patch (initially circular with radius $R=0.75$ ) in an anti-symmetric background flow through the gap (as in Fig. 2(b)) of strength, $\mathcal{F}=2 / \mathcal{S}$ for $a=1$. The strong shear forces the patch to split when close to the centre of the gap. Note the dotted line shows the point vortex trajectory starting at the same location as the patch. Snapshots taken at $t=0,70,108.5$.

\section{CONCLUSIONS}

Variation of the Rossby radius of deformation, $a$, affects trajectories of both point vortices and vortex patches near a gap in a barrier. In the absence of background flows, the most notable feature is that decrease in $a$ increases the tendency of a vortex to pass through a gap. Essentially this is due to the more rapid decay of the velocity field exterior to a vortex as $a$ decreases.

In a viscous fluid, separation and vorticity generation are expected near a sharp edge. For oceanographic applications, the gap edges are likely to be smoother and such separation may not be significant. In the absence of background flows, the most notable feature is that decrease in $a$ increases the tendency of a vortex to pass through a gap. Essentially this is due to the more rapid decay of the velocity field exterior to a vortex as $a$ decreases.

Using contour dynamics, vortex patches are compared to equivalent point vortices. The dynamics can also be normalised so as to give similar speeds and trajectories for vortex patches of varying size and point vortices in both the presence and absence of background flows at arbitrary values of $a$. In the limit that the patches remain circular, this normalisation is such that the speeds and trajectories are identical.

\section{ACKNOWLEDGMENTS}

R.S.N. is indebted to the UK Natural Environmental Research council for their support through their studentship Grant No. NE/F006454/1 and also acknowledges support from University College London (UCL) Department of Mathematics. 


\section{APPENDIX: ALTERNATIVE FORM OF THE INTEGRAL EQUATION AND ITS SOLUTION BY ITERATION}

In this Appendix, an alternative numerical method is described to solve (8)-(10). It is an iterative method based on perturbing about the exact solution for the infinite Rossby radius case. ${ }^{10}$

\section{Integral equation: Vortex sheet over the gap}

If the barrier is continuous, using the method of images, the velocity at the vortex can be found by placing an image vortex on the other side of the wall. The boundary conditions at the barriers are satisfied but not those at the gap. The influence of the gap can be retained by placing a vortex sheet over the gap with strength equal to the tangential velocity jump, $f(x)$, induced by the vortex and its image along the gap (see Ref. 10). Let $\psi(x, y)$ be the streamfunction induced by the vortex sheet across the gap and let $\psi(x, 0)=\Lambda(x)$ be its behaviour along the gap, $|x|<1, y=0$.

It is required to solve the boundary value problem for $\psi(x, y)$

$$
\begin{gathered}
\nabla^{2} \psi-\frac{1}{a^{2}} \psi=0, y>0, \\
\frac{\partial \psi}{\partial y}(x, 0)=f(x),|x|<1, \\
\psi(x, 0)=0,|x|>1, \\
\psi(r) \rightarrow 0 \text { as } r \rightarrow \infty, \text { where } r=\sqrt{x^{2}+y^{2}} .
\end{gathered}
$$

Here, $f(x)$ is the known function

$$
\begin{aligned}
f(x) & =\left.\frac{\partial}{\partial y}\left(\Psi_{P V}\left(x-x_{v}, y-y_{v}\right)-\Psi_{P V}\left(x-x_{v}, y+y_{v}\right)\right)\right|_{y=0} \\
& =\frac{-y_{v} K_{1}\left(\sqrt{\left(x-x_{v}\right)^{2}+y_{v}^{2}} / a\right)}{\pi a \sqrt{\left(x-x_{v}\right)^{2}+y_{v}^{2}}},
\end{aligned}
$$

where $x_{v}$ and $y_{v}$ are functions of time giving the vortex location. Let

$$
\begin{aligned}
G_{g}\left(x, y, x_{0}, y_{0}\right)= & -\frac{1}{2 \pi} K_{0}\left(\sqrt{\left(x-x_{0}\right)^{2}+\left(y-y_{0}\right)^{2}} / a\right) \\
& +\frac{1}{2 \pi} K_{0}\left(\sqrt{\left(x-x_{0}\right)^{2}+\left(y+y_{0}\right)^{2}} / a\right)
\end{aligned}
$$

be the Green function the upper half plane Dirichlet problem, i.e.,

$$
\begin{gathered}
\nabla^{2} G_{g}-\frac{1}{a^{2}} G_{g}=\delta\left(x-x_{0}\right) \delta\left(y-y_{0}\right), \\
G_{g}=0 \text { on } y=0 .
\end{gathered}
$$

Now, using Green's theorem and (A4)

$$
\iint_{S}\left(\psi \nabla^{2} G_{g}-G_{g} \nabla^{2} \psi\right) \mathrm{d} x \mathrm{~d} y=\int_{-\infty}^{\infty}\left(\psi \frac{\partial G_{g}}{\partial y}-G_{g} \frac{\partial \psi}{\partial y}\right) \mathrm{d} x,
$$

where $S$ is the upper half plane. 
Using (A1), (A8), and (A9), and $\psi(x, 0)=\Lambda(x)$ along the gap, the left hand side of (A10) may be simplified to give the following:

$$
\psi\left(x_{0}, y_{0}\right)=\int_{-1}^{1} \Lambda(x) \frac{\partial G_{g}}{\partial y}\left(x, 0, x_{0}, y_{0}\right) \mathrm{d} x .
$$

The velocity field at $\left(x_{0}, y_{0}\right)$ can be found by differentiating (A11) with respect to $x_{0}$ and $y_{0}$ to give the vertical and horizontal velocities, respectively,

$$
\begin{aligned}
& u\left(x_{0}, y_{0}\right)=-\int_{-1}^{1} \Lambda(x) \frac{\partial^{2} G_{g}}{\partial y \partial y_{0}}\left(x, 0, x_{0}, y_{0}\right) \mathrm{d} x, \\
& v\left(x_{0}, y_{0}\right)=\int_{-1}^{1} \Lambda(x) \frac{\partial^{2} G_{g}}{\partial y \partial x_{0}}\left(x, 0, x_{0}, y_{0}\right) \mathrm{d} x .
\end{aligned}
$$

Hence, given $\Lambda(x),|x| \leq 1$, the velocity field induced due to the vortex sheet can be computed everywhere using (A12) and (A13). In particular, by calculating the velocity at the vortex, located at $\left(x_{v}, y_{v}\right)$, the vortex can be advected and its trajectory can be found. It remains to find $\Lambda(x)$.

Differentiating (A11) with respect to $y_{0}$ and setting $y_{0}=0$ gives

$$
f\left(x_{0}\right)=\int_{-1}^{1} \Lambda(x) \frac{\partial^{2} G_{g}}{\partial y \partial y_{0}}\left(x, 0, x_{0}, 0\right) \mathrm{d} x .
$$

Since $f\left(x_{0}\right)$ is known, (A14) is an integral equation for the unknown $\Lambda(x)$. The numerical solution to the integral equation by iteration is discussed in Sec. 2 of this Appendix.

\section{Iterative solution}

For the vortex sheet over the gap, we can define the kernel function such that

$$
\mathcal{L}_{a}\left(x-x_{0}\right)=\frac{\partial^{2} G_{g}}{\partial y \partial y_{0}}\left(x, 0, x_{0}, 0\right) .
$$

Hence, the integral equation (A14) can be written as

$$
f\left(x_{0}\right)=\int_{-1}^{1} \Lambda(x) \mathcal{L}_{a}\left(x-x_{0}\right) \mathrm{d} x .
$$

Equation (A16) is a hypersingular integral equation since

$$
\mathcal{L}_{a}\left(x-x_{0}\right) \sim-\frac{1}{\pi\left(x-x_{0}\right)^{2}}+\mathrm{O}\left(x-x_{0}\right)^{-1}, \text { as } x \rightarrow x_{0} .
$$

Consider $G_{\infty}$, the rigid lid Green's function for Laplace's equation in the upper half plane,

$$
G_{\infty}=\frac{1}{4 \pi} \log \frac{\left(x-x_{0}\right)^{2}+\left(y-y_{0}\right)^{2}}{\left(x-x_{0}\right)^{2}+\left(y+y_{0}\right)^{2}} .
$$

Using the previous notation for the kernel function for the rigid lid case can be written as

$$
\mathcal{L}_{\infty}=-\frac{1}{\pi\left(x-x_{0}\right)^{2}} .
$$

This term can be introduced into the integral equation (A16), by adding and subtracting such that

$$
\int_{-1}^{1} \Lambda(x) \mathcal{L}_{\infty}\left(x-x_{0}\right) \mathrm{d} x=f\left(x_{0}\right)+\int_{-1}^{1} \Lambda(x)\left(\mathcal{L}_{\infty}\left(x-x_{0}\right)-\mathcal{L}_{a}\left(x-x_{0}\right)\right) \mathrm{d} x .
$$


Since the left hand side is an integral operator involving the rigid lid kernel function, it can be solved exactly if the right hand side is known. The iterative solution can be written as the following:

$$
\begin{aligned}
\int_{-1}^{1} \Lambda^{(n+1)}(x) \mathcal{L}_{\infty}\left(x-x_{0}\right) & =f\left(x_{0}\right)+\int_{-1}^{1} \Lambda^{(n)}(x)\left(\mathcal{L}_{\infty}\left(x-x_{0}\right)-\mathcal{L}_{a}\left(x-x_{0}\right)\right) \mathrm{d} x \\
& =f^{(n)}\left(x_{0}\right),
\end{aligned}
$$

where terms with superscripts $(n)$ are known and used to calculate the terms with superscripts $(n+1)$. All the integrations on the right hand side can be calculated numerically as the hypersingularities are subtracted from each other.

It remains to solve

$$
\int_{-1}^{1} \Lambda(x) \mathcal{L}_{\infty}\left(x-x_{0}\right)=f^{(n)}\left(x_{0}\right)
$$

which is equivalent to the rigid lid case, where $f^{(n)}\left(x_{0}\right)$ is half the vortex sheet strength and $\lambda^{(n+1)}(x)$ is the streamfunction along the gap. There is a direct method of computing this solution using Fourier transforms (see Ref. 10). In the present case, the solution is

$$
\Lambda^{(n+1)}(x)=\mathfrak{J} \sum_{k=1}^{\infty} \frac{b_{k}}{2 k} \zeta^{k},
$$

where $\zeta=x+i\left(1-x^{2}\right)$ and $b_{k}$ are the sine coefficients of the Fourier series of $f^{(n)}\left(x_{0}\right) . \Lambda(x)$ can be substituted into (A11) to give us the streamfunction and hence, the velocity field everywhere.

The method converges provided $a>1$ and vortices were farther than $\mathrm{O}(1)$ distance from the gap, otherwise numerical inaccuracies are encountered. In this case, it is preferable to consider vortex sheets placed over the semi-infinite barriers. When the iterative method converges, it produces identical trajectories to those computed by the method described in Sec. III.

${ }^{1}$ P. L. Richardson, A. S. Bower, and W. Zenk, "A census of Meddies tracked by floats," Prog. Oceanogr. 45, 209-250 (2000).

${ }^{2}$ H. L. Simmons and D. Nof, "The squeezing of eddies through gaps," J. Phys. Oceanogr. 32, 314 (2002).

${ }^{3}$ K. Jochumsen, M. Rhein, S. Hüttl-Kabus, and C. W. Böning, "On the propagation and decay of North Brazil current rings," J. Geophys. Res. 115, C10004, doi:10.1029/2009JC006042 (2010).

${ }^{4}$ W. Sheu, C. Wu, and L. Oey, "Blocking and westward passage of eddies in the Luzon Strait," Deep-Sea Res., Part II 57(19-20), 1783-1791 (2010).

${ }^{5}$ C. Cenedese, C. Adduce, and D. M. Fratantoni, "Laboratory experiments on mesoscale vortices interacting with two islands," J. Geophys. Res. 110, C09023, doi:10.1029/2004JC002734 (2005).

${ }^{6}$ J. Pedlosky, "Stratified abyssal flow in the presence of fractured ridges," J. Phys. Oceanogr. 24, 403 (1994).

${ }^{7}$ D. Nof, "Choked flows from the Pacific to the Indian Ocean," J. Phys. Oceanogr. 25, 1369 (1994).

${ }^{8}$ L. J. Pratt and M. A. Spall, "A porous media theory for barotropic flow through ridges and archipelagos," J. Phys. Oceanogr. 33, 2702-2718 (2003).

${ }^{9}$ P. G. Saffman, Vortex Dynamics (Cambridge University Press, Cambridge, 1992).

${ }^{10}$ E. R. Johnson and N. R. McDonald, "The motion of a vortex near a gap in a wall," Phys. Fluids 16, 462-469 (2004).

${ }^{11}$ E. R. Johnson and N. R. McDonald, "Vortices near barriers with multiple gaps," J. Fluid Mech. 531, 335-358 (2005).

${ }^{12}$ D. G. Crowdy and J. S. Marshall, "The motion of a point vortex around multiple circular islands," Phys. Fluids 17, 056602 (2005).

${ }^{13}$ M. Duran-Matute and O. U. Velasco Fuentes, "Passage of a barotropic vortex through a gap," J. Phys. Oceanogr. 38, 2817-2831 (2008).

${ }^{14}$ D. G. Dritschel, "Contour dynamics and contour surgery: Numerical algorithms for extended, high-resolution modelling of vortex dynamics in two-dimensional, inviscid, incompressible flows," Comp. Phys. Rep. 10, 77-146 (1989).

${ }^{15}$ J.-M. Vanden-Broeck and F. T. Smith, "Supercritical two-fluid interactions with surface tension and gravity," Mathematika 56, 93-106 (2010). 